\title{
Evaluation of coronary artery disease in congenital heart disease and pediatrics utilizing adenosine stress perfusion
}

\author{
Michael J Campbell, Piers CA Barker, Frederique Bailliard, Ray J Kim \\ From 2011 SCMR/Euro CMR Joint Scientific Sessions \\ Nice, France. 3-6 February 2011
}

\section{Introduction}

The diagnosis of CAD utilizing adenosine stress perfusion is well described in adults. CAD in children is rare but can occur in the setting of congenital and acquired heart disease. Adults with CHD, a growing patient population, are at risk for CAD. A review of the medical literature revealed one previous study involving 4 patients in which adenosine stress perfusion was used for evaluating CAD in these populations.

\section{Purpose}

To evaluate the safety and feasibility of adenosine stress perfusion in the assessment of coronary artery disease (CAD) in congenital heart disease (CHD) and pediatrics.

\section{Methods}

A retrospective chart review was performed on consecutive patients with a diagnosis of CHD or age $<18$ yo in which adenosine stress perfusion was attempted. SSFP cine and delayed enhancement CMR (DE-CMR) were performed in a standard manner. Adenosine stress perfusion was performed with the administration of 140ug/ $\mathrm{kg} / \mathrm{min}$ of adenosine for $2-4$ minutes and $0.1 \mathrm{mmol} / \mathrm{kg}$ of gadolinium using a standard adult protocol. Patients with abnormal DE-CMR in a pattern consistent with coronary artery distribution were considered to have myocardial infarction (MI). A stress perfusion defect larger than the infarct on DE-CMR was indicative of inducible ischemia.

Duke University, Durham, NC, USA

\section{Results}

32 studies were attempted in 30 patients (mean 31 years, $12<18$ years). $97 \%$ of the studies were completed safely and with images of diagnostic quality. Stress perfusion was discontinued in a patient with Ebstein's anomaly and atrial flutter who had increased ventricular rates with adenosine. General anesthesia was used in 3 studies. Diagnoses and symptoms are listed in Tables 1 and $2.35 \%$ of studies had evidence of CAD. $26 \%$ had DE-CMR evidence of MI. $16 \%$ had evidence of inducible

\section{Table 1 Diagnoses}

Tetralogy of Fallot $s / p$ repair

Kawasaki disease

Anomalous left coronary artery arising from pulmonary arteryrepaired

Ventricular septal defect $s / p$ repair

Coarctation of the aorta $s / p$ surgical repair

Coarctation of the aorta $s / p$ transcatheter stent

Left coronary artery arising from the right coronary sinus-repaired Right coronary artery arising from the left coronary sinus-repaired Anomalous right coronary artery arising from pulmonary arteryrepaired

Pulmonary atresia with intact ventricular septum

Transposition of the great arteries s/p Mustard operation

Right coronary artery aneurysm, etiology unknown

Aortic stenosis s/p Ross operation

Scimitar syndrome $s / p$ repair and $s / p$ coronary artery bypass graft Congenitally corrected transposition of the great arteries Hypoplastic right pulmonary artery, hypoplastic right coronary artery Dysplastic pulmonary valve with pulmonary insufficiency

Sinus venosus atrial septal defect and partial anomalous pulmonary venous return-repaired

Ebstein's anomaly s/p tricuspid valve repair . . 
Table 2 Presenting Symptom

\begin{tabular}{ll}
\hline Chest pain & 16 \\
\hline Asymptomatic, screening & 7 \\
\hline Abnormal echocardiogram & 3 \\
\hline Abnormal electrocardiogram & 2 \\
\hline Dyspnea & 2 \\
\hline Syncope & 1 \\
\hline Nausea and fatigue & 1 \\
\hline
\end{tabular}

ischemia on stress perfusion. 8 patients had a cardiac catheterization, and there was agreement with CMR in 6. A patient with repaired anomalous right coronary artery from the pulmonary artery had abnormal stress perfusion but normal catheterization. A patient with repaired anomalous left coronary artery from the pulmonary artery had a CMR in the post-operative period with evidence of infarct and inducible ischemia but no stenosis on catheterization. A CMR result negative for CAD resulted in no further workup in 18/20 (90\%). A finding of CAD on CMR resulted in continued workup or intervention in $9 / 11(82 \%)$.

\section{Conclusion}

CMR with adenosine stress perfusion can be safely performed in CHD and pediatrics. CMR can be used to evaluate $\mathrm{CAD}$ and influence outcomes in these populations.

Published: 2 February 2011

doi:10.1186/1532-429X-13-S1-P200

Cite this article as: Campbell et al:: Evaluation of coronary artery disease in congenital heart disease and pediatrics utilizing adenosine stress perfusion. Journal of Cardiovascular Magnetic Resonance 2011 13(Suppl 1): P200.
Submit your next manuscript to BioMed Central and take full advantage of:

- Convenient online submission

- Thorough peer review

- No space constraints or color figure charges

- Immediate publication on acceptance

- Inclusion in PubMed, CAS, Scopus and Google Scholar

- Research which is freely available for redistribution

Submit your manuscript at www.biomedcentral.com/submit
C Biomed Central 